\title{
Identification of Prime Factors to Maximize the Photocatalytic Hy- drogen Evolution of Covalent Organic Frameworks
}

\author{
Samrat Ghosh,* Akinobu Nakada, Maximilian A. Springer, Takahiro Kawaguchi, Katsuaki Suzuki, \\ Hironori Kaji, Igor Baburin, Agnieszka Kuc, Thomas Heine, Hajime Suzuki, Ryu Abe* and Shu Seki*
}

Supporting Information Placeholder

\begin{abstract}
Visible light driven hydrogen $\left(\mathrm{H}_{2}\right)$ production from water is a promising strategy to convert and store solar energy as chemical energy. Covalent organic frameworks (COFs) are front runners among different classes of organic photocatalysts. Photocatalytic activity of COFs depends on numerous factors such as electronic band gap, crystallinity, surface area, exciton migration, stability of transient species, charge separation and transport, etc. However, it is challenging to fine tune all these factors simultaneously to enhance the photocatalytic activity. Hence, in this report, an effort has been made to understand the interplay of these factors and identify the key factors for efficient photocatalytic $\mathrm{H}_{2}$ production through structure-property-activity relationship. Careful molecular engineering allowed us to optimize all the above plausible factors impacting on the overall catalytic activities of a series of isoreticular COFs. The present study determines three prime factors: light absorption, charge carrier generation, and their transport, which influence the photocatalytic $\mathrm{H}_{2}$ production of COFs in much greater extent than the other factors.
\end{abstract}

\section{INTRODUCTION}

Hydrogen is considered as one of the clean fuels, which can be produced using the inexhaustible sun light and copious water bodies by means of a suitable photocatalyst or photoelectrode. ${ }^{1-3}$ The very first report of such system came from Honda and Fujishima, who demonstrated an n-type $\mathrm{TiO}_{2}$ photoanode for water oxidation $\left(\mathrm{O}_{2}\right.$ production) coupled with $\mathrm{Pt}$ counter electrode for water reduction ( $\mathrm{H}_{2}$ production). ${ }^{4}$ Since then, researchers have intuitively studied every single element of the periodic table with the aim of developing new semiconductors, as photocatalysts or photoelectrodes with superior activity for water reduction and/or water oxidation. 3,5 However, till date, only a few of them have shown promising activity on a small scale and still needs to go a long way to meet the global energy need. ${ }^{6-8}$ Inspired by natural photosynthesis, chemists have started evaluating small molecular and polymeric organic photocatalysts for $\mathrm{H}_{2}$ and/or $\mathrm{O}_{2}$ production from water. Abundant functionalities and synthetic diversity of organic photocatalysts, allows to tune the light harvesting ability or band gaps, also they are easily processable, light weight and environmentally benign. ${ }^{9-12}$ The real potential of organic photocatalysts was realized when Wang, Domen and coworkers demonstrated relatively efficient $\mathrm{H}_{2}$ production from water by graphitic carbon nitride $\left(\mathrm{g}-\mathrm{C}_{3} \mathrm{~N}_{4}\right)$ loaded with Pt co-catalyst in the presence of a sacrificial electron donor (SED) in their pioneering work. ${ }^{13}$ Following this,
Cooper et. al. reported conjugated microporous polymers (CMPs), Thomas et. al. reported covalent triazine-based frameworks (CTFs) and Lotsch et. al. reported covalent organic frameworks (COFs) with improved light harvesting property and high surface area to achieve improved photocatalytic $\mathrm{H}_{2}$ production. ${ }^{14-18}$ Among these materials, recent studies have revealed that $\mathrm{COFs}$ exhibit superior photocatalytic $\mathrm{H}_{2}$ evolution, due to its crystalline and ordered mesoporous structure. ${ }^{18,19,20}$

COFs are one of the emerging next generation two- (2D) or three-dimensional (3D) polymeric materials, connected through reversible or irreversible covalent linkages. ${ }^{21-25}$ On demand chemical functionalization, tunable pore size, large surface area, high crystallinity and light weight make COFs a promising material for gas storage and separation, catalysis, energy storage, optoelectronics, sensing and drug delivery. ${ }^{26-}$ 31 In 2014, Lotsch and co-workers demonstrated $\mathrm{H}_{2}$ evolution from water using hydrazone-based COFs as photocatalyst loaded with Pt co-catalyst in presence of SED. ${ }^{32}$ Subsequently, a series of 2D COFs were reported from the same group and few others. ${ }^{33-40}$ Among these systems, a few exhibited very high $\mathrm{H}_{2}$ production, as comparable or higher to that of g$\mathrm{C}_{3} \mathrm{~N}_{4} .{ }^{37,39}$ These findings opened up new avenues for COFs to emerge as an efficient heterogeneous photocatalyst for $\mathrm{H}_{2}$ production.

Based on the available literature reports, we deduced the factors that affect the photocatalytic performance of COFs in different extent. These include light absorption (i.e. band gap), driving force for proton reduction, exciton migration, charge separation, charge carrier generation and transport, water dispersibility, stacking, crystallinity, surface area, morphology, stability of the transient species, linkage stability, proximity of SED and co-catalyst, as well as an interplay of all these factors. ${ }^{18,41}$ However, it is challenging to harmonize all the above mentioned factors at the same time to maximize the photocatalytic activity. Hence, it is crucial to identify the key factors, that affect the photocatalytic $\mathrm{H}_{2}$ evolution of COFs in greater extent than other factors, in order to design COFs with enhanced photocatalytic activity.

With this objective, herein, we have established a structureproperty-activity relationship for COFs, to recognize the prime factors for efficient photocatalytic $\mathrm{H}_{2}$ production. For this purpose, we have designed and synthesized a series of 2D isoreticular COFs by careful molecular engineering. Donoracceptor (D-A) conjugation, torsional angles, and reaction conditions were varied to realize the effect of light absorption, electronic band position, exciton migration, charge carrier generation and transport, layer stacking, crystallinity, surface 
Scheme 1. Chemical structures of the building blocks along with corresponding molecular lengths and torsional angles. Reaction scheme for the synthesis of COFs under different solvothermal conditions, condition A and condition B.

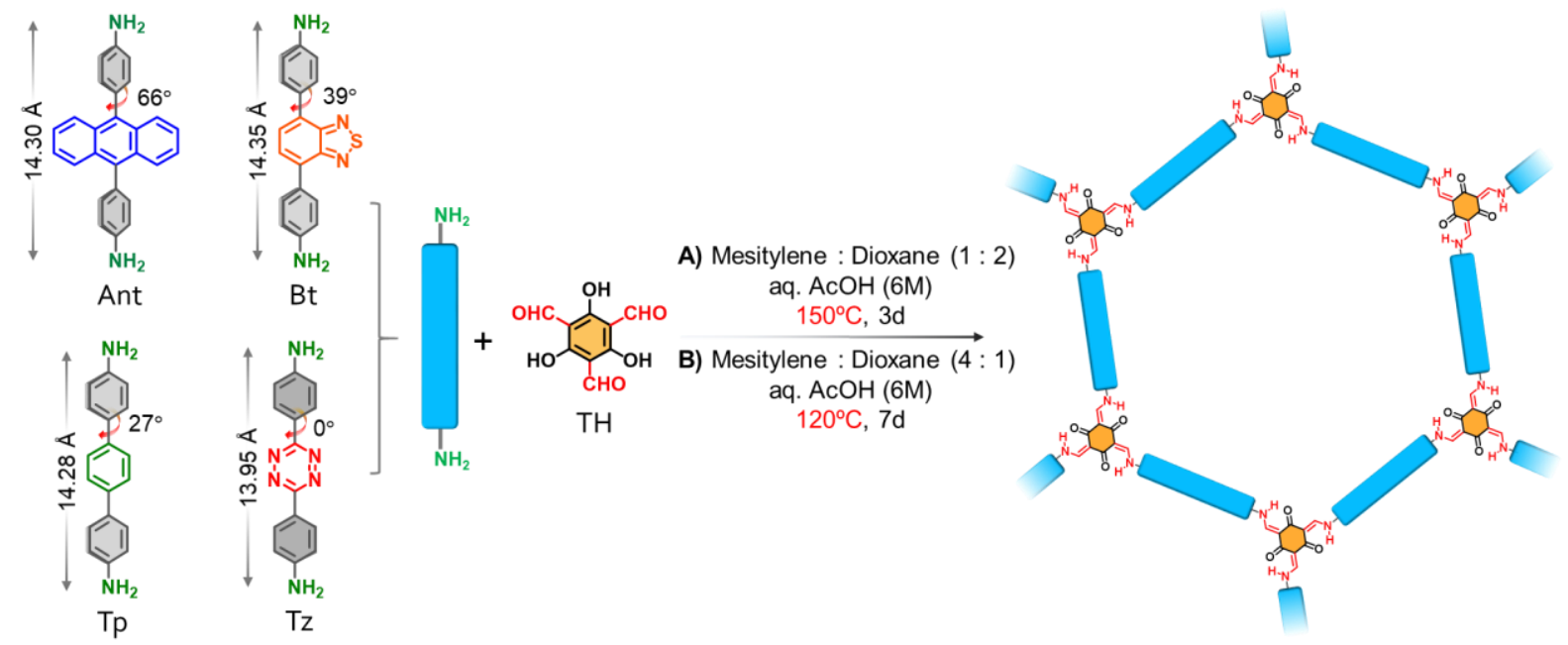

area and morphology over photocatalytic activity. Furthermore, the structure-property-activity relationship was extrapolated by generating mixed functional COFs. The photocatalytic activity of all these COFs was examined under visible light $(\geq 400 \mathrm{~nm})$ and have drawn a correlation to identify the prime factors, that would significantly affect the photocatalytic $\mathrm{H}_{2}$ evolution efficiency in these systems. Through our molecular design, high rate of $\mathrm{H}_{2}$ evolution $\left(750 \pm 25 \mu \mathrm{mol} \mathrm{g}{ }^{-1} \mathrm{~h}^{-1}\right)$ was achieved in the presence of low metallic Pt (1 wt\%) cocatalyst and triethanolamine as SED, with long-term stability and reusability.

\section{RESULTS AND DISCUSSION}

In order to generate $\beta$-ketoenamine linked isoreticular COFs, we have chosen 4,4" -diamino substituted $p$-terphenyl (Tp) and analogous derivatives as the linker, and condensed with 1,3,5-triformylphloroglucinol (TH) (Scheme 1). $\beta$ ketoenamine linked COFs are known to have very high chemical stability in water due to strong intramolecular $\mathrm{H}$-bonding which is desirable for photocatalytic applications. ${ }^{42-44}$ TpCOF was previously shown to be a photocatalyst for $\mathrm{H}_{2}$ evolution, and therefore, we have selected it as our reference to compare the photocatalytic activity of the newly synthesized COFs. ${ }^{38,39}$ However, it exhibited low photocatalytic activity, probably due to the low light absorption and wide band gap of $2.4 \mathrm{eV}$. To improve the light absorption, band gap, charge separation and transport, the central benzene ring of $\mathbf{T p}$ is substituted with electron rich anthracene (Ant) and electron deficient benzothiadiazole (Bt) and tetrazine $(\mathbf{T z}) \pi$-conjugated scaffold (Scheme 1). The other intention behind substitution is to tune the torsional angle of the central aryl ring with the peripheral phenyl rings to tune the surface area, crystallinity and stacking of the resulting COFs. Tp possess a torsional angle of $27^{\circ}$, which is increased to $66^{\circ}$ for Ant and $39^{\circ}$ in Bt due to the increase in $\mathrm{C}-\mathrm{H}$ steric repulsion between the peripheral phenyl rings and the $\alpha$ - proton of anthracene and $N$ - of benzothiadiazole, respectively (Scheme 1). Also, C-H steric repulsion diminishes upon replacing the central phenyl with $\mathrm{N}$-, resulting in a completely planar $\mathbf{T z}$. These torsional angles were estimated from the single crystal data reported previously. ${ }^{45-48}$
All the precursors were synthesized, purified, and characterized using high-resolution mass spectroscopy (HRMS), ${ }^{1} \mathrm{H}$ and ${ }^{13} \mathrm{C}$ nuclear magnetic resonance (NMR) (see Supporting Information). For the construction of $\beta$-ketoenamine linked COFs, acid-catalyzed solvothermal condensation was adopted (Scheme 1). TpCOF150 was synthesized according to the previous reports, ${ }^{39}$ using condition A (Scheme 1) and a similar condition was used to synthesize other isoreticular COFs, named as AntCOF150, BtCOF150 and TzCOF150 (Figure S1). Unfortunately, AntCOF150 exhibited low yield and crystallinity, hence, the reaction conditions were further optimized to obtain crystalline AntCOF by employing condition B (Scheme 1). Similarly, other COFs were synthesized using the same condition to improve the crystallinity and named as AntCOF120, BtCOF120, TPCOF120 and TzCOF120 (see Supporting Information).

Fourier transform infrared (FT-IR) spectra were recorded for all the COFs and compared with their corresponding building blocks (Figure S2). The disappearance of $-\mathrm{CHO}$ and $-\mathrm{NH}_{2}$ stretching frequencies and appearance of peaks at $\sim 1648 \mathrm{~cm}^{-}$ $1(\mathrm{C}=\mathrm{N})$ and $\sim 1257 \mathrm{~cm}^{-1}(\mathrm{C}-\mathrm{N})$ confirmed the quantitative polymerization. ${ }^{42,51}$ These COFs undergo keto-enol tautomerism and the equilibrium strongly depends on the reaction conditions such as solvent polarity, temperature, and amount of acid catalyst. ${ }^{42,51}$ Hence, the COFs synthesized using condition $\mathrm{A}$ and condition $\mathrm{B}$ may be expected to have different enol- and keto- content, however, difficult to assign from the FT-IR because of broad and merged stretching frequencies. To probe the presence of enol- and keto- form as well as intrinsic chemical connectivity, solid state ${ }^{13} \mathrm{C}$ cross polarization magic angle spinning (CP-MAS) NMR spectroscopy was carried out for all the COFs (Figure S3). By subtracting quaternary carbon spectra from all carbon, $-\mathrm{CH}$ carbon spectrum was obtained, which helps to distinguish. The assignment of carbons was confirmed by comparing with simulated ${ }^{13} \mathrm{C}$ NMR spectra of model compound using density functional theory (DFT, B3LYP/6-31G(d)). The absence of -CH carbon peak at around 180-200 ppm confirms the consumption of free -CHO of the precursor unit. Typically, the keto- form exhibits characteristic peak for carbonyl carbon and enamine carbon at 180-188 and 109 ppm, respectively, and both peaks were found to shift upfield in the enol form..$^{51}$ 

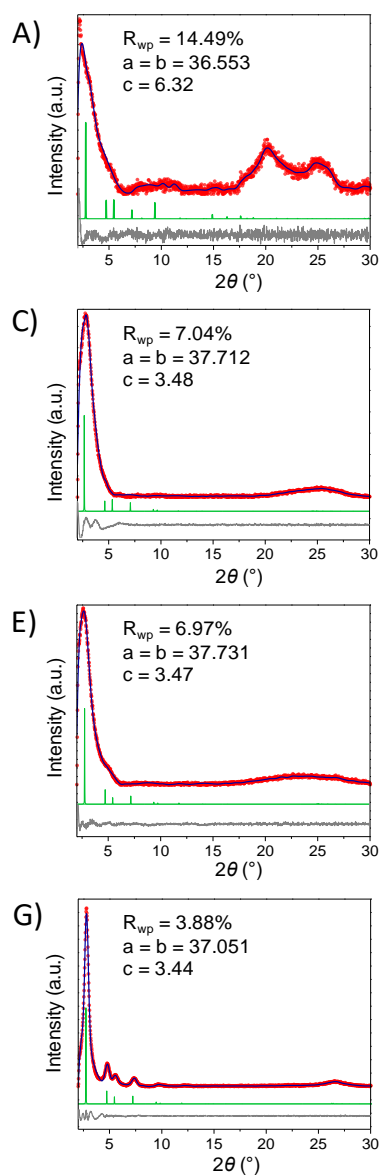
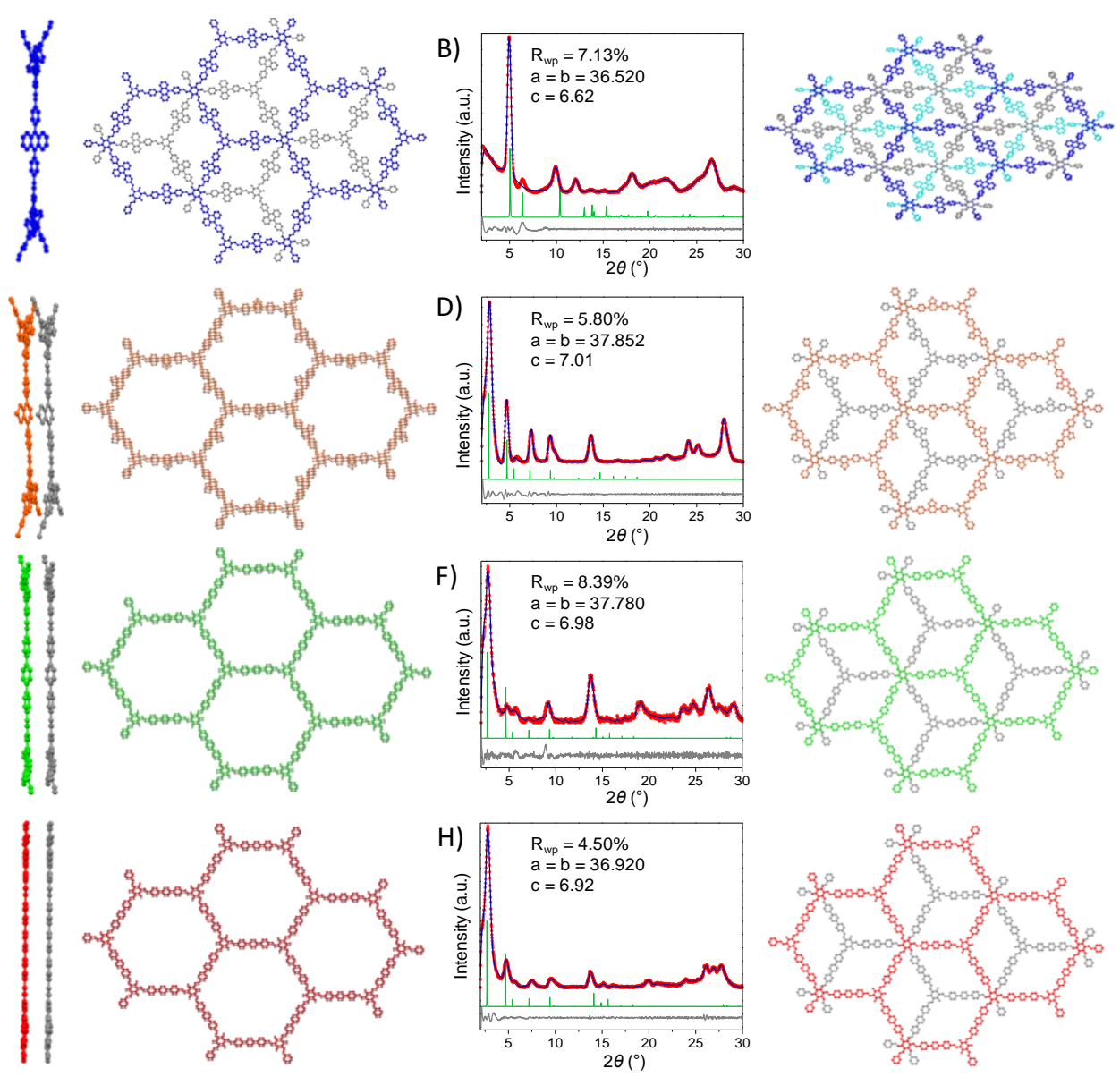

Figure 1. Experimental PXRD (red circle) and Pawley refined (blue) profiles of A) AntCOF150, B) AntCoF120, C) BtCoF150, D) BtCOF120, E) TpCOF150, F) TpCOF120, G) TzCOF150 and H) TzCOF120, with minimal differences between the experimental and the refined patterns (grey line). Simulated PXRD pattern (green line) and the side and top view of the plausible crystal structure of the corresponding stacking.

We then compared the ${ }^{13} \mathrm{C}$ CP-MAS NMR of the identical COFs synthesized under different conditions. COFs prepared at $150{ }^{\circ} \mathrm{C}$ showed sharp peak at $\sim 109 \mathrm{ppm}$, whereas emergence of a new peak at $\sim 102 \mathrm{ppm}$ was observed along with the peak at $\sim 109 \mathrm{ppm}$ for the COFs prepared at $120^{\circ} \mathrm{C}$ (Figure S3). This observation clearly indicates that the former COF is mostly composed of keto- form, while latter contains both keto- and enol- forms in varied proportions. This mean that COFs synthesized at $150{ }^{\circ} \mathrm{C}$ will have superior chemical stability due to intramolecular hydrogen bonding than COFs synthesized at $120^{\circ} \mathrm{C}$, which is desired for photocatalytic $\mathrm{H}_{2}$ evolution. ${ }^{38,39}$

In order to understand the effect of molecular torsional angle as well as the keto-enol tautomerism over molecular packing, powder X-ray diffraction (PXRD) analysis was performed for all the COFs (Figure 1). BtCOF150, TpCOF150 and TzCOF150 exhibited very similar PXRD pattern with an intense peak at $2 \theta=2.5 \sim 2.9^{\circ}$ along with few minor peaks. However, relatively low intensity broad peak was observed for AntCOF150 at $\sim 3^{\circ}$, probably due to the large torsional angle of Ant and high reaction temperature do not allow proper crystallization of 2D layers (Figure 1A)..$^{50}$ The last broad peak at $\sim 27.1^{\circ}$ in the PXRD patterns can be assigned to (001) plane resulting from the $\pi$-stacking of the 2D layers. Judging from the full width at half maximum (FWHM) of the first peak, the crystallinity as well as long range ordering increases upon decreasing the torsional angle of the building block (Figure S4). Surprisingly, by employing a slight change in reaction conditions, we have improved the crystallinity of AntCOF. AntCOF120 showed different PXRD pattern compared to AntCOF150 (Figure 1B). BtCOF120, TpCOF120 and TzCOF120 exhibits similar diffraction pattern, with most instance peak at $2.82^{\circ}, 2.73^{\circ}$ and $2.82^{\circ}$, respectively (Figure 1D, $\mathrm{F}$ and $\mathrm{H}$ ), along with several diffraction peaks at higher $2 \theta .^{47}$ We have compared the PXRD of COFs synthesized at $120{ }^{\circ} \mathrm{C}$ with their corresponding precursors and the absence of major diffraction peaks of the precursors in the diffractogram of the COFs, further confirms the absence of unreacted precursors in COFs (Figure S5). To elucidate the stacking of these COFs, three distinct stackings of 2D layers were considered, namely, $\mathrm{AA}^{\prime}, \mathrm{AB}$, and $\mathrm{ABC}$ (Figure 1). Optimized structures were obtained using density functional based tight- binding (DFTB+) method (further details are provided in Supporting Information). ${ }^{42,49}$ Similar torsional angles were observed for the optimized COF structures as that of 1 building blocks (Figure S6). Due to the high torsional angle of Ant, only $\mathrm{AB}$ and $\mathrm{ABC}$ stackings can be realized because of the steric hindrance. As a result, AntCOF120 in the ABC stacking is the energetically more favorable phase and the simulated PXRD pattern matches well with the experimental result (Figure 1B, Table S1). Also, the experimental PXRD patterns of BtCOF150, TpCOF150 and TzCOF150 are in a good agreement with the AA' stacking model, which corresponds to AA slipped stacking, where the layers are shifted with respect to each other by small offset (Figure 1C, E and G). ${ }^{49}$ 


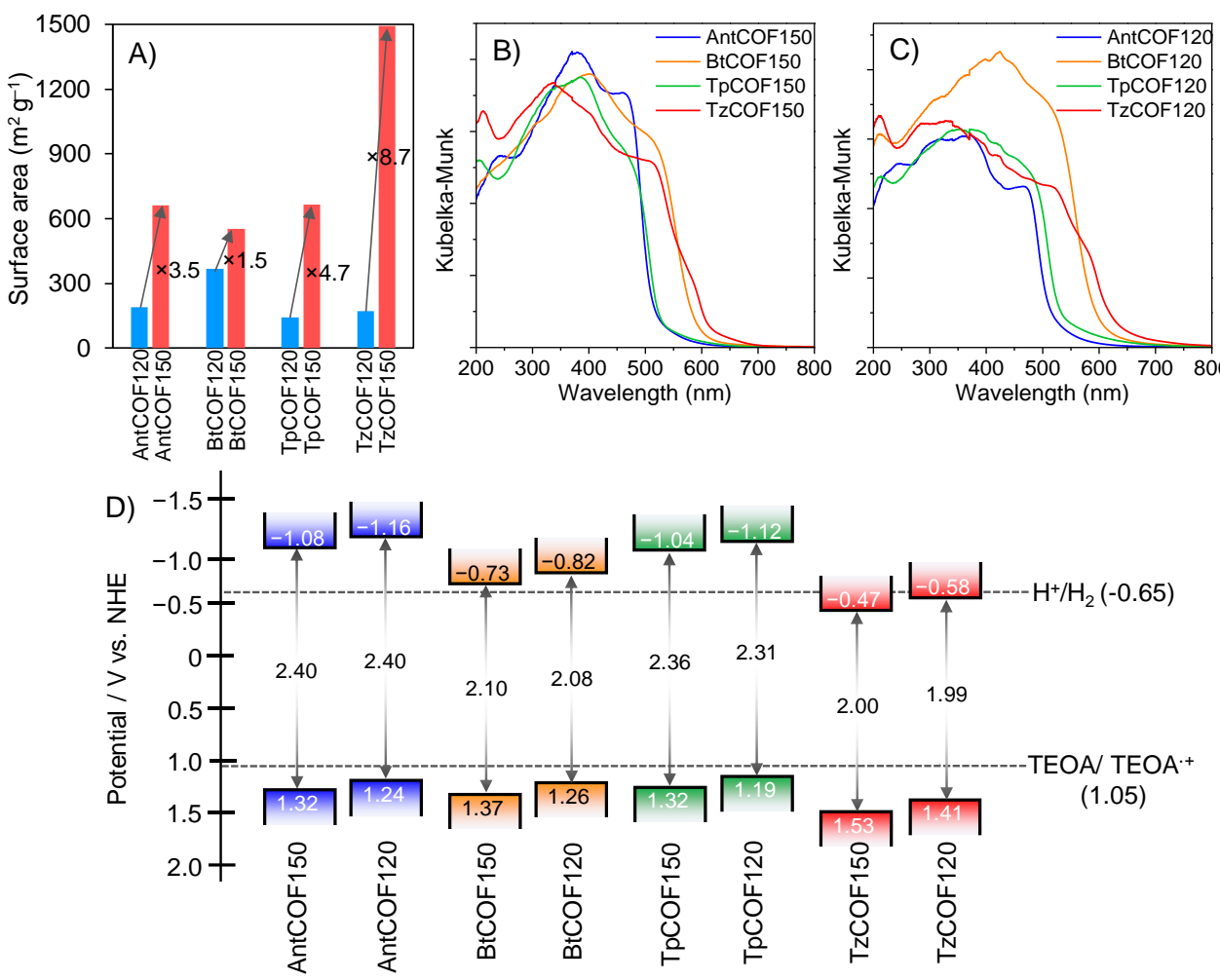

Figure 2. A) Comparison of the BET surface area of different COFs, calculated from nitrogen sorption isotherms at $77 \mathrm{~K}$. UV-Vis diffuse reflectance spectra of COFs B) prepared at $150{ }^{\circ} \mathrm{C}$ and $\mathrm{C}$ ) prepared at $120{ }^{\circ} \mathrm{C}$ in the solid state. D) Band position and bandgap of COFs vs normal hydrogen electrode (NHE), along with the potential of $\mathrm{H}^{+} / \mathrm{H}_{2}$ and TEOA/TEOA ${ }^{++}$at $\mathrm{pH}=11$.

We have considered $\mathrm{AA}^{\prime}$ compared to AA for these COFs, because relative stacking energy AA' lower than AA (Table S1). However, the low resolution of the PXRD does not allow us to distinguish various slipped conformations of $\mathrm{AA}^{\prime}$ stacked structures. On the other hand, the peak positions and relative intensities of BtCOF120, TpCOF120 and TzCOF120 fit well with the AB stacking model (Figure 1D, F and H). Finally, unit cell parameters of all the COFs were estimated by Pawley refinement of experimental PXRD with low residual value (Figure 1). The obtained unit cell parameters match well with the simulated cell parameters obtained from DFTB+.

Permanent porosities of all these COFs were evaluated by nitrogen adsorption-desorption analysis at $77 \mathrm{~K}$. Most of these COFs exhibited type-I isotherm as evident from the adsorption curves, which is a characteristic of microporous materials (Figure S7). Only TzCOF150 displayed type-IV isotherm, indicating mesoporous framework (Figure S7d). The Brunauer-Emmett-Teller (BET) surface areas of COFs synthesized at $150{ }^{\circ} \mathrm{C}$ were found to always be higher than those synthesized at $120{ }^{\circ} \mathrm{C}$, due to the preferred AA' stacking as evident from PXRD (Figure 2A, Table S2). The BET surface areas of the four COFs were determined as $660 \mathrm{~m}^{2} \mathrm{~g}^{-1}$ (AntCOF150), $554 \quad \mathrm{~m}^{2} \quad \mathrm{~g}^{-1}$ (BtCOF150), $665 \mathrm{~m}^{2} \quad \mathrm{~g}^{-1}$ (TpCOF150) and $1491 \mathrm{~m}^{2} \mathrm{~g}^{-1}$ (TzCOF150). As expected, an increase in the BET surface area was observed upon decreasing the torsional angles of the linkers and TzCOF150 showed maximum surface area because of the planer structure of the Tz. ${ }^{33}$ However, AntCOF150 showed slightly higher surface area than BtCOF150 and TpCOF150, probably due to the presence of amorphous microporous polymeric phase. Nonlocal density functional theory (NLDFT) revealed that the pore size distribution of COFs synthesized at $120^{\circ} \mathrm{C}$ is smaller than COFs prepared at $150{ }^{\circ} \mathrm{C}$ (Figure S7, Table S2) and experi- mental pore size matches with the theoretically predicted pore size. Hence, we have generated two sets of COFs from the same building blocks, having different crystallinity, stacking arrangement and surface area, to realize the structureproperty relationship over photocatalytic $\mathrm{H}_{2}$ evolution.

The phase purity of all the COFs were established from the scanning electron microscopy (SEM) images. SEM images revealed that all the COFs exhibit unique single morphology, that confirms the phase purity of the sample (Figure S8). Also, the high temperature $\left(150^{\circ} \mathrm{C}\right)$ COFs resulted in smaller mesostructured COFs than the low temperature $\left(120^{\circ} \mathrm{C}\right)$ ones, which may help to disperse themselves better in water, leading to an increase in the available catalytic sites. Thermal stability of all the COFs were analyzed using thermogravimetric analysis (TGA) under nitrogen atmosphere and these COFs exhibited excellent thermal stability up to $400{ }^{\circ} \mathrm{C}$ except TzCOF, which start to decompose at $250{ }^{\circ} \mathrm{C}$ (Figure S9).

Prior to photocatalytic studies, the light harvesting properties and band gaps of these COFs were assessed using UV-Vis diffuse reflectance spectra (Figure 2B and C). Both AntCOF150 and TpCOF150 exhibited relatively narrow optical absorbance with the absorption onset at $520 \mathrm{~nm}$, direct optical bandgap of $2.4 \mathrm{eV}$ (Figure 2B). However, BtCOF150 and TzCOF150 showed more than $100 \mathrm{~nm}$ red shift in absorption onset with a direct optical bandgap of $2.0 \mathrm{eV}$. Such red shifted broad absorption with lower optical band gap arises from the conjugated D-A structures where benzothiadiazole or tetrazine act as electron acceptors. The COFs prepared from the same ligand at different synthetic conditions showed similar absorption profile (Figure 2C). The highest occupied molecular orbitals (HOMOs) of all the COFs were estimated using photo yield spectroscopy (PYS) (Figure S10) and lowest unoccupied molecular orbitals (LUMOs) were calculated from 
the optical band gaps and the HOMO energy levels (Figure 2D). To gain more insights, the frontier molecular orbitals and energy levels were calculated for the smallest repeating unit of AntCOF, BtCOF, TpCOF and TzCOF. Structural optimizations and HOMO-LUMO energy levels were calculated using DFT (B3LYP-/6-31G*). A gradual lowering in LUMO energy levels was observed with the increase in acceptor strength (Figure S11), which is in line with the experimentally obtained positive shift in the LUMO potential (Figure 2D). The estimated band gaps are 3.26, 2.74, 3.32 and $2.91 \mathrm{eV}$ for AntCOF, BtCOF, TpCOF and TzCOF respectively, which are larger than the experimental band gaps because we have considered the smallest unit without stacking. However, the DFT calculated energy levels and band gaps fairly match with the experimentally observed trends.

Based on LUMO energy levels, it is evident that all the synthesized COFs have sufficient driving force for proton reduction except TzCOF and the driving force drastically decreases with an increase in the acceptor strength (Figure 2D). The significant positive potential shift of LUMO in TzCOF originates from the low-lying $\pi^{*}$ orbital of tetrazine. ${ }^{52}$ Hence, in principle, all the newly synthesized COFs have the ability to liberate $\mathrm{H}_{2}$ photocatalytically, except TzCOF. Next, photocatalytic $\mathrm{H}_{2}$ production activity of all these COFs were examined under visible light $(\geq 400 \mathrm{~nm}$ ) in the presence of Pt co-catalyst for reducing the overpotential of proton reduction and triethanolamine (TEOA) as SED to regenerate the photocatalyst by capturing the photogenerated holes. The photocatalytic activity of all the COFs were carried out under similar conditions for effective comparison of their efficiency. As expected, a steady $\mathrm{H}_{2}$ evolution was detected under visible light illumination $(\geq 400 \mathrm{~nm})$ for all the COFs except TzCOF in the test period of $24 \mathrm{~h}$ (Figure 3A). Despite having a broad light absorption, high surface area and crystallinity, TzCOF showed no evolution of $\mathrm{H}_{2}$ due to insufficient driving force for proton reduction (Figure 2D). Interestingly, BtCOF150 exhibited very high photocatalytic $\mathrm{H}_{2}$ evolution rate among the other COFs with a marginal driving force for proton reduction. In general, better photocatalytic activity was observed for COFs synthesized at $150{ }^{\circ} \mathrm{C}$ rather than at $120^{\circ} \mathrm{C}$. Average $\mathrm{H}_{2}$ evolution rates were calculated at the end of $24 \mathrm{~h}$ photocatalysis and found to be $55 \pm 5 \mu \mathrm{mol} \mathrm{h}-1 \mathrm{~g}^{-1}, 50 \pm 4 \mu \mathrm{mol} \mathrm{h}^{-1} \mathrm{~g}^{-1}$ and $750 \pm 25 \mu \mathrm{mol} \mathrm{h} \mathrm{h}^{-1} \mathrm{~g}^{-1}$ for AntCOF150, TpCOF150 and BtCOF150, respectively (Figure 3B). On the other hand, poor $\mathrm{H}_{2}$ production was observed for AntCOF120, TpCOF120 and BtCOF120 with a rate of $16 \pm 2 \mu \mathrm{mol} \mathrm{h}^{-1} \mathrm{~g}^{-1}, 16 \pm 2 \mu \mathrm{mol} \mathrm{h}^{-1} \mathrm{~g}^{-1}$ and $95 \pm 7 \mu \mathrm{mol} \mathrm{h}^{-1} \mathrm{~g}^{-1}$, respectively. None of the COFs produced $\mathrm{H}_{2}$ in the absence of Pt catalyst. The photocatalytic activity of BtCOF150 in presence of low amount of Pt (1 wt\%) is comparable with the benchmark systems such as $\mathrm{Pt}$-modified amorphous melon $\left(720 \mu \mathrm{mol} \mathrm{h}^{-1} \mathrm{~g}^{-1}\right)^{53}$ or 'g-C3N4' $(840 \mu \mathrm{mol}$ $\left.\mathrm{h}^{-1} \mathrm{~g}^{-1}\right)^{54}$ or crystalline poly(triazineimide) $\left(864 \mu \mathrm{mol} \mathrm{h} \mathrm{h}^{-1} \mathrm{~g}^{-}\right.$ 1). ${ }^{53}$ BtCOF150 showed 15.1 fold higher rate of $\mathrm{H}_{2}$ evolution compared to TpCOF150, whereas with respect to the same TpCOF, the previously reported TPBDDACOF and FSCOF exhibited 13.2 times and 6.4 times $\mathrm{H}_{2}$ evolution rate, respectively (Figure S12). ${ }^{38,39}$ The amount of Pt was varied to improve the photocatalytic activity of BtCOF150 and found saturation in the photocatalytic activity with 1 wt \% Pt (Figure $\mathrm{S} 13 \mathrm{a}$ ). Also, the apparent quantum yield (AQY) for BtCOF150, was found to be $0.2 \%$ at $420 \mathrm{~nm}$ which is comparable to ' $\mathrm{g}$ C3N4'.13 Long term photocatalytic activity of BtCOF150 was examined and no significant deactivation was observed for $120 \mathrm{~h}$, indicating excellent durability and recyclability (Figure S14). After $120 \mathrm{~h}$ of photocatalysis, almost $1.7 \mathrm{mmol}$ of $\mathrm{H}_{2}$ was obtained, which exceeds the hydrogen contents of BtCOF150 used for the photocatalysis. This result clearly suggest that $\mathrm{H}_{2}$ does not come from the COF decomposition. ${ }^{55-57}$ Also, we have investigated the stability of all the COFs using FT-IR, ${ }^{13} \mathrm{C}$ NMR, and PXRD after $24 \mathrm{~h}$ of photocatalysis. FT-IR revealed the retention of molecular connectivity for all the COFs (Figure S15). Furthermore, chemical stability of the ligand and the linkers were attested by the ${ }^{13} \mathrm{C}$ CP-MAS and ${ }^{15} \mathrm{~N}$ NMR measurements (Figure S16). However, a partial loss in the long range ordering was observed for most of the COFs in different extent as evident from PXRD measurements (Figure S17). Also, photocatalysis does not lead to any major morphological transformation in any of the developed COFs, as evident from the SEM and high-resolution transmission electron microscope (HR-TEM) analysis (Figure S18 and S19). In addition, TEM images of post-photocatalysis COF samples revealed the deposition of platinum nanoparticle (below $10 \mathrm{~nm}$ ) over the COF surface (Figure S19e-h).

In order to prioritize the key factors for efficient $\mathrm{H}_{2}$ evolution, we have correlated the photocatalytic activity of all the COFs with the torsional angle, surface area, crystallinity, stacking, light absorption, band gap, driving force for proton reduction and photoconductivity in the following section (Table 1). AntCOF120 possesses higher crystallinity compared to AntCOF150 but showed $\sim 3.5$ times reduced rate of $\mathrm{H}_{2}$ production. This can be correlated with the $\sim 3.5$ times decrease in surface area for AntCOF120 compared to AntCOF150, since both the COFs have same light harvesting ability and band position (Figure 2B-D). In this case, we have not considered the effect of charge transport, because AntCOF120 and AntCOF150 prefers $A B C$ and $A B$ stacking, where interlayer chromophore distance is more than $6 \AA$, which is not suitable for charge transport. Similarly, TpCOF120 has better light absorption and driving force for proton reduction than TpCOF150, however the three times higher rate of $\mathrm{H}_{2}$ production in the latter can be attributed to the increase in surface area ( $\sim 4.7$ times) as well as AA' stacking. BtCOF150 exhibited enhanced photocatalytic activity (15.1 times) than TpC0F150 despite having reduced driving force for the proton reduction, comparable crystallinity and surface area (Table 1). Probably, in this case, the broad light absorption (i.e. low band gap) and better charge carrier generation and transport of D-A conjugated BtCOF150 plays an important role when compared to other factors (Table 1). To estimate the effect of light absorption, wavelength dependent $\mathrm{H}_{2}$ evolution measurements were performed for all the COFs using different band-pass filters and compared with the UV-Vis spectra. Both BtCOF120 and BtCOF150 showed $\mathrm{H}_{2}$ evolution activity up to $600 \mathrm{~nm}$, while negligible $\mathrm{H}_{2}$ evolution was observed for the other COFs beyond $550 \mathrm{~nm}$ (Figure S13b and S13c). To our surprise, BtCOF150 showed eight fold increased rate of $\mathrm{H}_{2}$ evolution compared to BtCOF120, although both have comparable crystallinity, light absorption, bandgap and the driving force for proton reduction. Hence, it is clear that such an increase in the photocatalytic activity arises from the cumulative effect of increase in surface area, stacking and charge carrier mobility. However, the surface area increase was only 1.5 times (Figure 2A), which clearly state that stacking and charge transport has a major say in the increase in photocatalytic activity. 

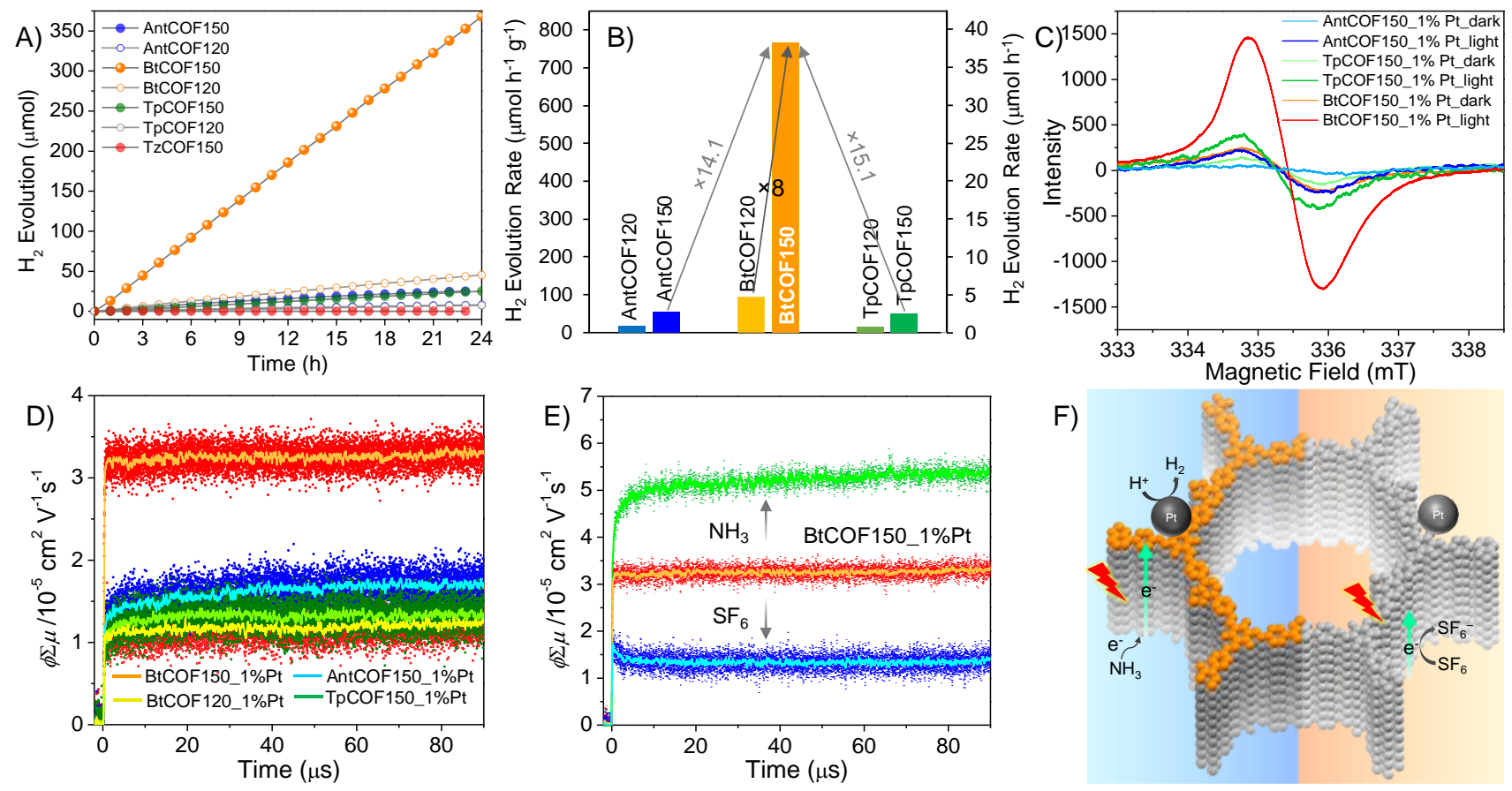

Figure 3. A) Time course for photocatalytic $\mathrm{H}_{2}$ production using visible light ( $\geq 400 \mathrm{~nm}$ ) of all the prepared COFs (20 mg COF, 1 wt \% Pt, water-TEOA $(4: 1,100 \mathrm{ml})$ ). B) Comparison of photocatalytic hydrogen evolution rates. C) EPR spectra of AntCOF150, TpCOF150 and BtCOF150 loaded with $1 \mathrm{wt} \% \mathrm{Pt}$, under light and dark conditions. D) Transient photoconductivities of BtCOF150, BtCOF120, TpCOF150 and AntCOF150 loaded with 1 wt\% Pt. E) FP-TRMC transients of BtCOF150_1\%Pt under N2 (red), NH3 (green) and $\mathrm{SF}_{6}$ (blue) atmosphere. F) Schematic representation of the plausible processes after the photoexcitation of the BtCOF150 under the $\mathrm{NH}_{3}$ (light blue) and $\mathrm{SF}_{6}$ (light red) atmosphere.

To support the above-mentioned notion, the photoconductivity of all the COFs was measured using flash-photolysis time-resolved microwave conductivity (FP-TRMC). FP-TRMC is a powerful electrodeless method to access the intrinsic/local charge transporting property of semiconductors, that provides a measure of photoconductivity as $\phi \Sigma \mu$, where $\phi$ and $\Sigma \mu$ corresponds to the charge carrier generation quantum yield and the total charge carrier mobilities (electron + hole mobilities), respectively. ${ }^{58-61}$ Negligible photoconductivity was observed for all the pristine COFs upon $355 \mathrm{~nm}$ laser excitation, probably due to a very small charge separation. A significant enhancement in photoconductivity was observed for the COFs loaded with $1 \mathrm{wt} \%$ Pt nanoparticle because of the photogenerated charge separation and transport (Figure 3D). Maximum photoconductivity of $3.2 \times 10^{-5} \mathrm{~cm}^{2} \mathrm{~V}^{-1} \mathrm{~s}^{-1}$ was observed for the BtCOF150_1\%Pt, which is 3 times higher than the BtCOF120_1\%Pt, indicating the improvement in $\Sigma \mu$ rather than $\phi$, because both COFs carry the same chromophore. All the kinetic traces of the photoconductivity transients show long lifetimes over $100 \mu$ s, suggesting the significant contribution from the free charge carriers. Furthermore, to discern the charge carriers (hole or electron), photoconductivity was measured under the $\mathrm{NH}_{3}$ (hole scavenger) and $\mathrm{SF}_{6}$ (electron scavenger) atmosphere. Interestingly, BtCOF150_1\%Pt exhibited an increase in the value of under $\mathrm{NH}_{3}$ whereas a decrease in $\phi \Sigma \mu$ was observed under $\mathrm{SF}_{6}$ atmosphere (Figure 3E). ${ }^{62} \mathrm{NH}_{3}$ which is chemically analogous to TEOA, acts as an electron donor and transfer an electron to the photoexcited BtCOF150, leading to enhanced charge separation and electron transport, resulting in an increase in $\phi \Sigma \mu$ (Figure 3F). On the other hand, decrease in $\phi \Sigma \mu$ indicates the trapping of mobile electrons by $\mathrm{SF}_{6}$. These results clearly reveal the efficient electron transporting ability of BtCOF150, creating an easy path for the transport of electrons to the platinum center for proton reduction.

To have a theoretical support, band structures were calculated for the $\mathrm{AA}^{\prime}$, and $\mathrm{AB}$ stacked BtCOF, within $k_{\mathrm{B}} T\left(k_{\mathrm{B}}\right.$, Boltzmann constant) at room temperature, both are directgap semiconductors with calculated bandgap of $1.3 \mathrm{eV}$. These band gaps are smaller than the experimentally measured ones, which is as expected, because DFTB tends to underestimate them. Interestingly, AA' stacked structure exhibits strong dispersion in the conduction band edge compared to the $A B$ stacked BtCOF (Figure S20). As a result, the effective masses of electrons are smaller for the AA' stacking, possibly leading to higher charge carrier mobility (Table S3). ${ }^{63}$ These results clearly corroborate with the trends in charge carrier mobilities obtained from the FP-TRMC measurement. On the other hand, AntCOF150 and TpCOF150 exhibited similar photoconductivity as BtCOF120, although latter exhibited slightly better photocatalytic activity, because of broad light absorption (Table 1). The photoconductivity results are in line with the observed $\mathrm{H}_{2}$ evolution activity of the COFs. These results confirm that the intrinsic charge carrier mobility is one of the prime factors that can greatly affect the photocatalytic $\mathrm{H}_{2}$ evolution efficiency. Moreover, light induced charge carrier generation was revealed using another microwave based technique, electron paramagnetic resonance (EPR) spectroscopy. BtCOF150_1\%Pt exhibited a signal at $g=2.004$ which intensifies upon visible light excitation, indicating charge carrier generation (Figure 3C). Compared to BtCOF150_1\%Pt, all other COFs showed very low intensity in presence of light, due to the reduced generation of charge carriers. 
Table 1. Torsional angles, surface area, crystallinity, photophysical properties and the rate of $\mathrm{H}_{2}$ evolution of all the COFs.

\begin{tabular}{|c|c|c|c|c|c|c|c|c|c|}
\hline COF & $\begin{array}{c}\text { Torsional } \\
\text { angle }\left(^{\circ}\right)\end{array}$ & $\begin{array}{c}\text { BET } \\
\text { Surface } \\
\text { area } \\
\left(\mathrm{m}^{2} \mathrm{~g}^{-1}\right)\end{array}$ & $\begin{array}{c}\text { Degree of Crys- } \\
\text { tallinity }\end{array}$ & Stackinga & $\begin{array}{c}\text { Light ab- } \\
\text { sorption } \\
\text { area }\left(\mathrm{nm}^{2}\right)^{\mathrm{b}}\end{array}$ & $\begin{array}{c}\text { Bandgap } \\
(\mathrm{eV})^{\mathrm{c}}\end{array}$ & $\begin{array}{c}\text { Driving } \\
\text { force } \\
(\mathrm{V})^{\mathrm{d}}\end{array}$ & $\begin{array}{c}\mathrm{H}_{2} \text { evolu- } \\
\text { tion rate } \\
\left(\mu \mathrm{mol} \mathrm{g}^{-1} \mathrm{~h}^{-}\right. \\
1)^{\mathrm{e}}\end{array}$ & $\begin{array}{c}\varphi \Sigma \mu \\
\left(10^{-5}\right. \\
\mathrm{cm}^{2} \mathrm{~V}^{-1} \mathrm{~s}^{-} \\
1)^{\mathrm{f}}\end{array}$ \\
\hline AntCOF120 & 66 & 191 & Crystalline & $\mathrm{ABC}$ & 92.5 & 2.40 & -0.51 & $16 \pm 2$ & 0.5 \\
\hline AntCOF150 & 66 & 660 & Amorphous & $\mathrm{AB}$ & 91.8 & 2.40 & -0.43 & $55 \pm 5$ & 1.2 \\
\hline BtCOF120 & 39 & 368 & Crystalline & $\mathrm{AB}$ & 147.6 & 2.08 & -0.17 & $95 \pm 7$ & 1.0 \\
\hline BtCOF150 & 39 & 554 & Semicrystalline & $\mathrm{AA}^{\prime}$ & 134.8 & 2.10 & -0.08 & $750 \pm 25$ & 3.2 \\
\hline TpCOF120 & 27 & 141 & Semicrystalline & $\mathrm{AB}$ & 108.5 & 2.31 & -0.47 & $16 \pm 2$ & 0.6 \\
\hline TpCOF150 & 27 & 665 & Semicrystalline & $\mathrm{AA}^{\prime}$ & 89.7 & 2.36 & -0.39 & $50 \pm 4$ & 1.1 \\
\hline TzCOF150 & 0 & 1491 & Crystalline & $\mathrm{AA}^{\prime}$ & 142.7 & 2.00 & 0.18 & 0 & 0.2 \\
\hline
\end{tabular}

aCalculated from theoretical simulation. bNormalized between 400-800 $\mathrm{nm}$. cEstimated from absorption onset. dDriving force for proton reduction $=\left(\right.$ LUMO -0.65 (potential of $\left.\left.\mathrm{H}^{+} / \mathrm{H}_{2}\right)\right) \mathrm{V}$ vs NHE at $\mathrm{pH}=11$. eAll the hydrogen evolution rates were measured using

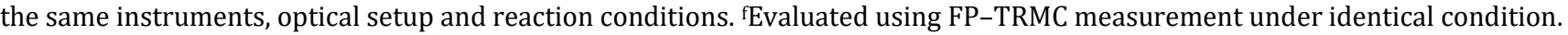

Also, we have performed the same experiment for the pristine COFs (without Pt) and again BtCOF150 showed relatively intense signal compared to AntCOF150 and TpCOF150 (Figure S22). It is noteworthy to mention that in general Pt loaded COFs showed higher signal intensities compared with the pristine COFs upon light illumination, indicating charge separation between COFs and Pt co-catalyst. These results are in line with the photoconductivity data obtained from the FPTRMC measurement.

Finally, we have done the bandgap engineering of the COFs to enhance the light harvesting property and charge separation and understand their effects on the photocatalytic $\mathrm{H}_{2}$ evolution. Our unique molecular design has allowed us to create mixed or doped COFs due to very similar linker length and geometry (Scheme 1). Moreover, we have the freedom to modulate the surface area and crystallinity using this approach. This can further lead us to extrapolate the structureproperty-activity relationship, which can validate our hypothesis. For this, we have incorporated Bt linker in the frameworks of AntCOF, TpCOF and TzCOF to boost their photocatalytic activity (Figure 4A). BtAntCOF, BtTpCOF and BtTzCOF was synthesized by taking the corresponding building blocks (1:1) and condensed with $\mathbf{T H}$ in equimolar ratio using condition A (further details are provided in Supporting Information). Formation of mixed COFs were confirmed using FT-IR, ${ }^{13} \mathrm{C}$ CP-MAS NMR and PXRD. FT-IR and ${ }^{13} \mathrm{C}$ CP-MAS NMR revealed the chemical structure of the mixed COFs and these COFs mostly exists as keto-form, due to the absence of peak at $\sim 102 \mathrm{ppm}$ corresponding to the -enol form (Figure S23). BtAntCOF showed better crystallinity compared to AntCOF150 due to the introduction of low torsional angle of Bt which rigidifies the framework (Figure S24a). Similarly, insertion of $\mathbf{T p}$ and $\mathbf{T z}$ linkers with lower torsional angle than Bt, enhances the crystallinity of BtTpCOF and BtTzCOF significantly, compared with BtCOF150 (Figure S24b and S24c). Mixed COFs structure were simulated by considering equal number of two different building blocks in unit cell, for re- spective COFs. Similar to the previous section, three distinct stackings of $2 \mathrm{D}$ layers were considered, namely, $\mathrm{AA}$ ', $\mathrm{AB}$ and ABC. PXRD of the mixed COFs exhibited greater similarity with the simulated AA' stacking (Figure S24d-f). Also, Pawley refinement fit well with the experimental PXRD having low residual value for all the mixed COFs, testify ing the lattice parameters (Figure S24g-i). BtAntCOF and BtTpCOF showed better surface area than the individual COFs, whereas decrease in surface area was observed for BtTzCOF (Figure S25). All the mixed COFs showed color change, red shifted broad absorption compared to higher band gap COFs and shift in HOMO energy, confirm mixing of the linkers (Figure 4A, Figure S26). Also, mixing leads to a significant decrease in the direct optical band gap from $2.4 \mathrm{eV}$ to $2.1 \mathrm{eV}$ for both BtAntCOF and BtTpCOF, whereas for BtTzCOF, the band gap remains unchanged compared to individual COFs (Figure 4BC, Figure S27).

Next, we have checked the photocatalytic $\mathrm{H}_{2}$ production activity of all the mixed COFs under the same conditions as used for the individual COFs (Figure S28). Figure 4D shows a comparative plot of the amount of $\mathrm{H}_{2}$ produced during a test period of $24 \mathrm{~h}$. BtTpCOF produced maximum $\mathrm{H}_{2}$ among other mixed COFs, whereas BtAntCOF showed slight improvement compared to AntCOF150 and still no $\mathrm{H}_{2}$ evolution was observed for BtTzCOF. Mixing leads to $\sim 9$ times improvement in photocatalytic activity for BtTpCOF compared to TpCOF150, with a $\sim 1.8$ times increase in surface area and decreased crystallinity, confirming the key role of light harvesting property to improve photocatalytic activity. However, despite having higher surface area, moderate crystallinity and similar light absorption, $\sim 7$ and $\sim 1.6$ times lower photocatalytic activity was observed for BtAntCOF and BtTpCOF compared to BtCOF150 (Table S4). In order to have a comparison, we have physically mixed equal amounts (1:1 wt ratio) of AntCOF150 with BtCOF150 and TpCOF150 with BtCOF150 and both the physically mixed COFs exhibited superior $\mathrm{H}_{2}$ production compared to chemically mixed BtAntCOF and BtTpCOF, however 

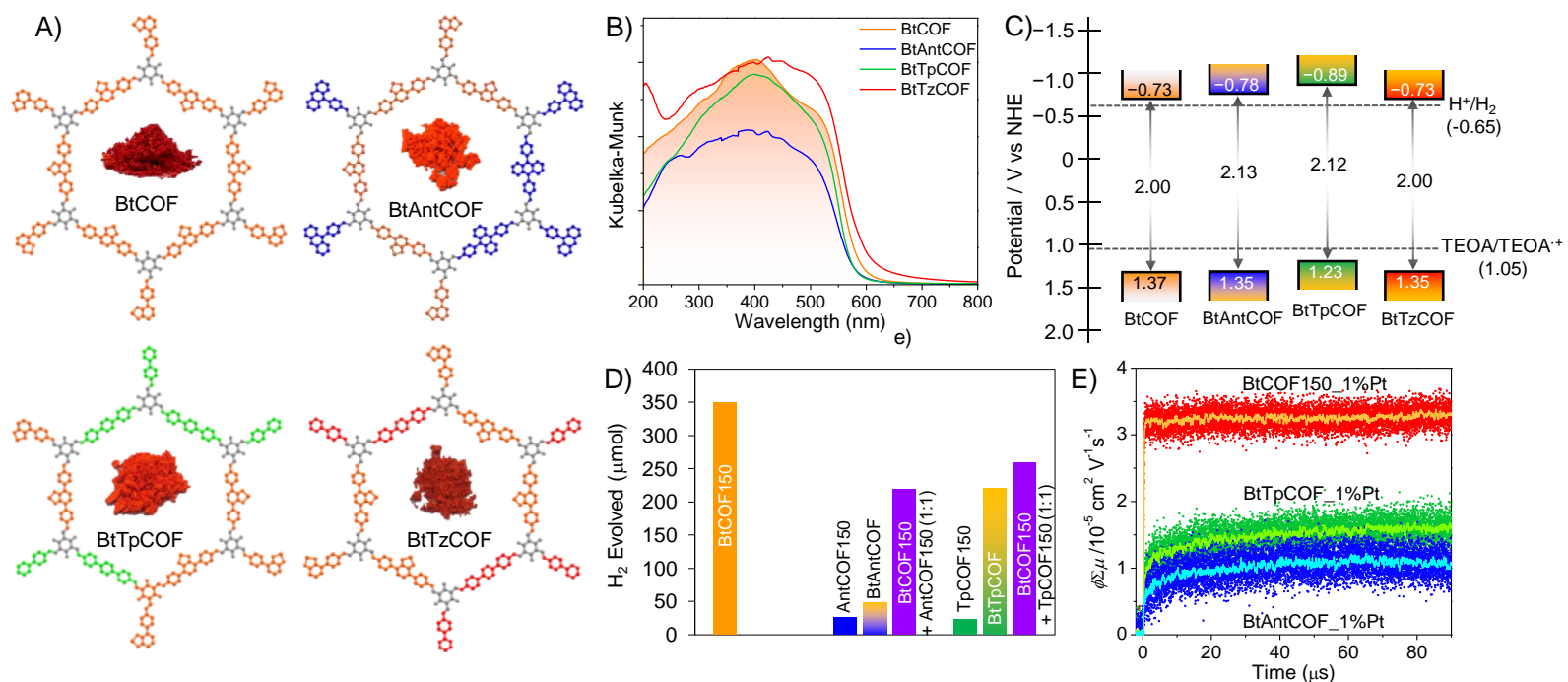

Figure 4. A) Structures of mixed COFs along with the photographs of the COF powders under ambient light. B) UV-Vis diffuse reflectance spectra of the mixed COFs in the solid state. C) Band position and bandgap of mixed COFs vs NHE, along with the potential of $\mathrm{H}^{+} / \mathrm{H}_{2}$ and TEOA/TEOA ${ }^{+}$at $\mathrm{pH}=11$. D) Comparison of photocatalytic $\mathrm{H}_{2}$ production of mixed COFs with the individual COFs under visible light irradiation after $24 \mathrm{~h}$. E) Transient photoconductivities of BtCOF150, BtAntCOF and BtTpCOF loaded with 1 wt $\%$ Pt.

$\sim 1.6$ and $\sim 1.3$ times lower photocatalytic activity compared to BtCOF150. Theoretically, one can expect 2 times or lower photocatalytic $\mathrm{H}_{2}$ production for both chemically and physically mixed COFs than BtCOF150 because of 2 times reduction in the amount of BtCOF150. These results point to the random arrangement of linkers inside the chemically mixed COFs, which drastically decreases the charge transporting property. To precisely probe the charge carrier generation and transport, we have checked the photoconductivity of mixed COFs by FP-TRMC and compared with BtCOF150 (Figure $4 \mathrm{E}$ ). Both the mixed COFs showed much lower photoconductivity compared to BtCOF150, thus suggesting that the conductive path of BtCOF150 was blocked by the random arrangement of chromophores. Also, it can be deduced that high torsional angle of Ant causes larger distance between the chromophores of BtAntCOF. On the other hand, lower torsional angle of $\mathbf{T p}$ offers shorter chromophoric distance, that keep some conductive paths open in BtTpCOF resulting in moderate $\mathrm{H}_{2}$ production. After photocatalysis, all the mixed COFs were well characterized by FT-IR, ${ }^{13} \mathrm{C}$ NMR, and PXRD. These experimental results confirmed the chemical and structural robustness of mixed COFs during the photocatalysis (Figure S29-30). Moreover, morphological analyses such as SEM and HR-TEM was performed for the pre- and post- photocatalysis samples of mixed COFs, which confirmed the retention in morphology and the deposition of platinum nanoparticles over COF surface (Figure S31-32).

\section{CONCLUSION}

In summary, we have designed and synthesized novel isoreticular 2D COFs via systematic molecular engineering of the building blocks, that leads to a gradual change in porosity, crystallinity, stacking, optical and electronic properties. As a result, these COFs exhibited different photocatalytic $\mathrm{H}_{2}$ production under visible light and we have successfully correlated their photocatalytic activity with the above-mentioned factors. We have observed that light absorption, charge carrier generation and their transport has greater influence on the photocatalytic $\mathrm{H}_{2}$ production, compared to other factors. Also, it is important to mention that, $\mathrm{AA}^{\prime}, \mathrm{AB}$ and $\mathrm{ABC}$ stacked COFs all are crystalline, but our results indicate that AA' stacking exhibits better photocatalytic activity among others because it dramatically improves the exciton migration and charge transport. As a result, BtCOF150 showed maximum rate of $\mathrm{H}_{2}$ evolution (750 $\left.\pm 25 \mu \mathrm{mol} \mathrm{g}^{-1} \mathrm{~h}^{-1}\right)$ among all the developed COFs, in presence of low metallic Pt (1 wt $\%)$ co-catalyst, comparable to $\mathrm{g}-\mathrm{C}_{3} \mathrm{~N}_{4}$ and benchmark COFs reported previously. ${ }^{18}$ Furthermore, unlike microporous polymers a negative effect on the $\mathrm{H}_{2}$ evolution was observed upon introducing dissimilar linkers into BtCOF150, although there was an improvement in optical properties. ${ }^{14}$ This finding again manifests the immense role of charge carrier mobility over other factors. All the results are well supported by the photoconductivity measurement (FP-TRMC), EPR and DFTB calculations. The structure-property-activity correlation presented here will pave the way to the design of highly efficient COF photocatalyst with enhanced activity.

\section{ASSOCIATED CONTENT}

\section{Supporting Information}

The Supporting Information is available free of charge on the ACS Publications website. Details of materials synthesis, characterization, structural simulation and photocatalysis (PDF).

\section{AUTHOR INFORMATION}

\section{Corresponding Authors}

Samrat Ghosh - Department of Molecular Engineering, Graduate School of Engineering, Kyoto University, Nishikyo-ku, Kyoto 615-8510, Japan; orcid.org/0000-0002-5946-3013; Email: samratghosh8888@gmail.com

Ryu Abe - Department of Energy and Hydrocarbon Chemistry, Graduate School of Engineering, Kyoto University, Nishikyo-ku, Kyoto 615-8510, Japan; orcid.org/0000-0001-8592-076X; Email: ryu-abe@scl.kyoto-u.ac.jp

Shu Seki - Department of Molecular Engineering, Graduate School of Engineering, Kyoto University, Nishikyo-ku, Kyoto 
615-8510, Japan; orcid.org/0000-0001-7851-4405; Email: seki@moleng.kyoto-u.ac.jp

\section{Authors}

Akinobu Nakada - Department of Energy and Hydrocarbon Chemistry, Graduate School of Engineering, Kyoto University, Nishikyo-ku, Kyoto 615-8510, Japan; orcid.org/0000-00016670-5044; Email: nakada.773@g.chuo-u.ac.jp

Maximilian A. Springer - Theoretical Chemistry, Technische Universität Dresden, Bergstr. 66c, 01062 Dresden, Germany; Helmholtz-Zentrum Dresden-Rossendorf, Institut für Ressourcenökologie, Forschungsstelle Leipzig, Permoserstr. 15, 04318 Leipzig, Germany; Email: m.springer@hzdr.de

Takahiro Kawaguchi - Department of Molecular Engineering, Graduate School of Engineering, Kyoto University, Nishikyo-ku, Kyoto 615-8510, Japan; Email: takahirokawaguchi0523@gmail.com

Katsuaki Suzuki - Institute for Chemical Research, Kyoto University, Uji, Kyoto, 611-0011, Japan; Email: suzuki.katsuaki.8a@kyoto-u.ac.jp

Hironori Kaji - Institute for Chemical Research, Kyoto University, Uji, Kyoto, 611-0011, Japan; orcid.org/0000-0002-51113852; Email: kaji@scl.kyoto-u.ac.jp

Igor Baburin - Faculty for Chemistry and Food Chemistry, Bergstrasse 66c, 01069 Dresden, Germany; Email: Igor.Baburin@chemie.tu-dresden.de

Agnieszka Kuc - Theoretical Chemistry, Technische Universität Dresden, Bergstr. 66c, 01062 Dresden, Germany; HelmholtzZentrum Dresden-Rossendorf, Institut für Ressourcenökologie, Forschungsstelle Leipzig, Permoserstr. 15, 04318 Leipzig, Germany; Email: a.kuc@hzdr.de

Thomas Heine - Theoretical Chemistry, Technische Universität Dresden, Bergstr. 66c, 01062 Dresden, Germany; HelmholtzZentrum Dresden-Rossendorf, Institut für Ressourcenökologie, Forschungsstelle Leipzig, Permoserstr. 15, 04318 Leipzig, Germany; orcid.org/0000-0003-2379-6251; Email: thomas.heine@tu-dresden.de

Hajime Suzuki - Department of Energy and Hydrocarbon Chemistry, Graduate School of Engineering, Kyoto University, Nishikyo-ku, Kyoto 615-8510, Japan; orcid.org/0000-00028891-2033; Email: suzuki.hajime.7x@kyoto-u.ac.jp

\section{Notes}

The authors declare no competing financial interests.

\section{ACKNOWLEDGMENT}

This work was partly supported by a Grant-in-Aid for Scientific Research (no. 26102011, 15K21721, 17H06439, $19 F 19044$ and 18H03918) from the Japan Society for the Promotion of Science (JSPS). M. S., A. K., I. B, and T. H. thank the high-performance computing centre, ZIH Dresden, for computer time and recourses, and Deutsche Forschungsgemeinschaft for financial support (SPP 1928, HE 3543/31-1). It is our pleasure to thank Prof. Koichi Eguchi for allowing T.K. to perform the nitrogen isotherm. We thank Prof. Hiroki Kurata and Mr. Tsutomu Kiyomura for the HR-TEM analysis. Dr. Y. Tsutsui, Dr. T. Sakurai and Dr. W. Masuda are acknowledged for the fruitful discussions.

\section{REFERENCES}

(1) Kudo, A.; Miseki, Y. Heterogeneous photocatalyst materials for water splitting. Chem. Soc. Rev. 2009, 38, 253-278.

(2) Pinaud, B. A.; Benck, J. D.; Seitz, L. C.; Forman, A. J.; Chen, Z.; Deutsch, T. G.; James, B. D.; Baum, K. Technical and economic feasibility of centralized facilities for solar hydrogen production via photocatalysis and photoelectrochemistry. Energy Environ. Sci. 2013, 6, 1983-2002.

(3) Wang, Y.; Suzuki, H.; Xie, J.; Tomita, O.; Martin, D. J.; Higashi, M.; Kong, D.; Abe, R.; Tang, J. Mimicking natural photosynthesis: solar to renewable $\mathrm{H}_{2}$ fuel synthesis by Z-scheme water splitting systems. Chem. Rev. 2018, 118, 5201-5241.

(4) Fujishima, A.; Honda, K. Electrochemical photolysis of water at a semiconductor electrode. Nature 1972, 238, 37-38.

(5) Chen, S.; Takata, T.; Domen, K. Particulate photocatalysts for overall water splitting. Nat. Rev. Mater. 2017, 2, 17050

(6) Liao, L.; Zhang, Q.; Su, Z.; Zhao, Z.; Wang, Y.; Li, Y.; Lu, X.; Wei, D.; Feng, G.; Yu, Q. Efficient solar water-splitting using a nanocrystalline CoO photocatalyst. Nat. Nanotechnol. 2014, 9, 69-73.

(7) Kibria, M. G.; Chowdhury, F. A.; Zhao, S.; AlOtaibi, B.; Trudeau, M. L.; Guo, H.; Mi, Z. Visible light-driven efficient overall water splitting using p-type metal-nitride nanowire arrays. Nat. Commun. 2015, 6 6797.

(8) Wang, Q.; Hisatomi, T.; Jia, Q.; Tokudome, H.; Zhong, M.; Wang, C.; Pan, Z.; Takata, T.; Nakabayashi, M.; Shibata, N.; Li, Y.; Sharp, I. D.; Kudo, A.; Yamada, T.; Domen, K. Scalable water splitting on particulate photocatalyst sheets with a solar-to-hydrogen energy conversion efficiency exceeding 1\%. Nat. Mater. 2016, 15, 611-615 (2016).

(9) Yanagida, S.; Kabumoto, A.; Mizumoto, K.; Pac, C.; Yoshino, K. Poly(para) phenylene-catalyzed photoreduction of water to hydrogen Chem. Commun. 1985, 8, 474-475.

(10) Caputo, C. A.; Gross, M. A.; Lau, V. W.; Cavazza, C.; Lotsch, B. V.; Reisner, E. Photocatalytic hydrogen production using polymeric carbon nitride with a hydrogenase and a bioinspired synthetic $\mathrm{Ni}$ catalyst. Angew. Chem. Int. Ed. 2014, 53, 11538-11542.

(11) Liu, Y.; Liu, N.; Han, Y.; Zhang, X.; Huang, H.; Lifshitz, Y.; Lee, S.-T.; Zhong, J.; Kang, Z. Metal-free efficient photocatalyst for stable visible water splitting via a two-electron pathway. Science $\mathbf{2 0 1 5}$ 347, 970-974.

(12) Zhang, G.; Lan, Z.-A.; Wang, X. Conjugated polymers: catalysts for photocatalytic hydrogen evolution. Angew. Chem. Int. Ed. 2016, 55, 2-18.

(13) Wang, X.; Maeda, K.; Thomas, A.; Takanabe, K.; Xin, G.; Carlsson, J. M.; Domen, K.; Antonietti, M. A metal-free polymeric photocatalyst for hydrogen production from water under visible light. Nat. Mater. 2009, 8, 76-80.

(14) Sprick, R. S.; Jiang, J. X.; Bonill, o B.; Ren, S.; Ratvijitvech, T.; Guiglion, P.; Zwijnenburg, M. A.; Adams, D. J.; Cooper, A. I. Tunable organic photocatalysts for visible-light-driven hydrogen evolution. J. Am. Chem. Soc. 2015, 137, 3265-3270.

(15) Kuhn, P.; Antonietti, M.; Thomas, A. Porous, covalent triazine-based frameworks prepared by ionothermal synthesis. Angew. Chem. Int. Ed. 2008, 47, 3450-3453.

(16) Jiang, X.; Wang, P.; Zhao, J. 2D covalent triazine framework: a new class of organic photocatalyst for water splitting. Journal of Materials Chemistry A 2015, 3, 7750-7758.

(17) Kuecken, S.; Acharjya, A.; Zhi, L.; Schwarze, M.; Schomacker, R.; Thomas, A. Fast tuning of covalent triazine frameworks for photocatalytic hydrogen evolution. Chem. Commun. 2017, 53, 5854-5857.

(18) Banerjee, T.; Gottschling, K.; Savasci, G.; Ochsenfeld, C. Lotsch, B. V. $\mathrm{H}_{2}$ evolution with covalent organic framework photocatalysts. ACS Energy Lett. 2018, 3, 400-409.

(19) Vyas, V. S.; Lau, V. W. H.; Lotsch, B. V. Soft photocatalysis: organic polymers for solar fuel production. Chem. Mater. 2016, 28 5191-5204.

(20) Thote, J.; Aiyappa, H. B.; Deshpande, A.; Diaz, D. D.; Kurungot, S.; Banerjee, R. A covalent organic framework-cadmium sulfide hybrid as a prototype photocatalyst for visible-light-driven hydrogen production. Chem. Eur. J. 2014, 20, 15961-15965.

(21) Côté, A. P.; Benin, A. I.; Ockwig, N. W.; Keeffe, M.; Matzger, A. J.; Yaghi, O. M. Porous, Crystalline, Covalent Organic Frameworks. Science 2005, 310, 1166-1170.

(22) Feng, X.; Ding, X. S.; Jiang, D. Covalent organic frameworks. Chem. Soc. Rev. 2012, 41, 6010-6022.

(23) Colson, J. W.; Dichtel, W. R. Rationally synthesized twodimensional polymers. Nat. Chem. 2013, 5, 453.

(24) Diercks, C. S.; Yaghi, O. M. The atom, the molecule, and the covalent organic framework. Science 2017, 355, eaal1585.

(25) Lohse, M. S.; Bein, T. Covalent organic frameworks: structures, synthesis, and applications. Adv. Funct. Mater. 2018, 28, 1705553. 
(26) Waller, P. J.; Gándara, F.; Yaghi, O. M. Chemistry of Covalent Organic Frameworks. Acc. Chem. Res. 2015, 48 (12), 3053-3063.

(27) Huang, N.; Wang, P.; Jiang, D. Covalent organic frameworks: a materials platform for structural and functional designs. Nat. Rev. Mater. 2016, 1, 16068.

(28) Segura, J. L.; Mancheño, M. J.; Zamora, F. Covalent organic frameworks based on schiff-base chemistry: synthesis, properties and potential applications. Chem. Soc. Rev. 2016, 45, 5635-5671.

(29) Mandal, A. K.; Mahmood, J.; Baek, J. B. Two-dimensional covalent organic frameworks for optoelectronics and energy storage. ChemNanoMat 2017, 3, 373-391.

(30) Beuerle, F.; Gole, B. Covalent organic frameworks and cage compounds: design and applications of polymeric and discrete organic scaffolds. Angew. Chem., Int. Ed. 2018, 57, 4850-4878.

(31) Song, Y.; Sun, Q.; Aguila, B.; Ma, S. Opportunities of Covalent Organic Frameworks for Advanced Applications. Adv. Sci. 2019, 6, 1801410

(32) Stegbauer, L.; Schwinghammer, K.; Lotsch, B. V. A hydrazonebased covalent organic framework for photocatalytic hydrogen production. Chem. Sci. 2014, 5, 2789-2793.

(33) Vyas, V. S.; Haase, F.; Stegbauer, L.; Savasci, G.; Podjaski, F.; Ochsenfeld, C.; Lotsch, B. V. A tunable azine covalent organic framework platform for visible light-induced hydrogen generation. Nat. Commun. 2015, 6, 8508.

(34) Haase, F.; Banerjee, T.; Savasci, G.; Ochsenfeld, C.; Lotsch, B. V. Structure-property-activity relationships in a pyridine containing azine-linked covalent organic framework for photocatalytic hydrogen evolution. Faraday Discuss. 2017, 162, 165-169.

(35) Banerjee, T.; Haase, F.; Savasci, G.; Gottschling, K.; Ochsenfeld, C.; Lotsch, B. V. Single site photocatalytic $\mathrm{H}_{2}$ evolution from covalent organic frameworks with molecular cobaloxime co-catalysts. J. Am. Chem. Soc. 2017, 139, 16228-16234.

(36) Sick, T.; Hufnagel, A. G.; Kampmann, J.; Kondofersky, I.; Calik, M.; Rotter, J. M.; Evans, A.; Doblinger, M.; Herbert, S.; Peters, K.; Bohm D.; Knochel, P.; Medina, D. D.; Fattakhova-Rohlfing, D.; Bein, T. Oriented films of conjugated 2D covalent organic frameworks as photocathodes for water splitting. J. Am. Chem. Soc. 2018, 140, 2085-2092.

(37) Bi, S.; Yang, C.; Zhang, W.; Xu, J.; Liu, L.; Wu, D.; Wang, X.; Han Y.; Liang, Q.; Zhang, F. Two-dimensional semiconducting covalent organic frameworks via condensation at arylmethyl carbon atoms. Nat. Commun. 2019, 10 (1), 2467.

(38) Pachfule, P.; Acharjya, A.; Roeser, J.; Langenhahn, T.; Schwarze, M.; Schomacker, R.; Thomas, A.; Schmidt, J. Diacetylene functionalized covalent organic framework (COF) for photocatalytic hydrogen generation. J. Am. Chem. Soc. 2018, 140, 1423-1427.

(39) Wang, X.; Chen, L.; Chong, S. Y.; Little, M. A.; Wu, Y.; Zhu, W.-H.; Clowes, R.; Yan, Y.; Zwijnenburg, M. A.; Sprick, R. S.; Cooper, A. I. Sulfone-containing covalent organic frameworks for photocatalytic hydrogen evolution from water. Nat. Chem. 2018, 10, 1180-1189.

(40) Jin, E.; Lan, Z.; Jiang, Q.; Geng, K.; Li, G.; Wang, X.; Jiang, D. 2D $\mathrm{sp}^{2}$ Carbon-Conjugated Covalent Organic Frameworks for Photocatalytic Hydrogen Production from Water. Chem. 2019, 5 (6), 1632-1647.

(41) Wang, Y.; Vogel, A.; Sachs, M.; Sprick, R. S.; Wilbraham, L.; Moniz, S. J.; Godin, R.; Zwijnenburg, M. A.; Durrant, J. R.; Cooper, A. I. Current understanding and challenges of solar-driven hydrogen generation using polymeric photocatalysts. Nat. Energy 2019, 4, 1-15.

(42) Kandambeth, S.; Mallick, A.; Lukose, B.; Mane, M. V.; Heine, T.; Banerjee, R. Construction of crystalline 2D covalent organic frameworks with remarkable chemical (acid/base) stability via a combined reversible and irreversible route. J. Am. Chem. Soc. 2012 134, 19524-19527.

(43) Biswal, B. P.; Chandra, S.; Kandambeth, S.; Lukose, B.; Heine, T.; Banerjee, R. Mechanochemical Synthesis of Chemically Stable Isoreticular Covalent Organic Frameworks. J. Am. Chem. Soc. 2013, 135, 5328-5331.

(44) Kandambeth, S.; Biswal, B. P.; Chaudhari, H. D.; Rout, K. C.; Kunjattu H., S.; Mitra, S.; Karak, S.; Das, A.; Mukherjee, R.; Kharul, U. K. Banerjee, R. Selective Molecular Sieving in Self-Standing Porous Covalent-Organic-Framework Membranes. Adv.Mater. 2017, 29, 1603945.

(45) Rietveld, H. M.; Maslen E. N. An X-ray and neutron diffraction refinement of structure of para-terphenyl. Acta Crystallogr 1970, B26, 693-706.

(46) Kato, S.; Matsumoto, T.; Shigeiwa, M.; Gorohmaru, H.; Maeda S.; Ishi-i, T.; Mataka, S. Novel 2,1,3-benzothiadiazole-based red- fluorescent dyes with enhanced two-photon sbsorption cross sections. Chem.-Eur. J. 2006, 12, 2303-2317.

(47) Langer, V.; Becker, H.-D. Crystal structure of 9,10diphenylanthracene, $\left(\mathrm{C}_{6} \mathrm{H}_{5}\right)\left(\mathrm{C}_{14} \mathrm{H}_{8}\right)\left(\mathrm{C}_{6} \mathrm{H}_{5}\right)$. Z. Kristallogr., 1992, 199, 313315.

(48) Ahmed, N. A.; Kitaigorodsky, A. I. Experimental and theoretical determination of the crystal structure of 3,6-diphenyl-s-tetrazine. Acta Crystallogr. 1972, B28, 739-742.

(49) Lukose, B.; Kuc, A.; Heine, T. The structure of layered covalent-organic frameworks. Chem. Eur. J. 2010, 17, 2388-2392.

(50) Thompson, C. M.; Occhialini, G.; McCandless, G. T.; Alahakoon, S. B.; Cameron, V.; Nielsen, S. O.; Smaldone, R. A. Computational and experimental studies on the effects of monomer planarity on covalent organic framework formation. J. Am. Chem. Soc. 2017, 139, 10506-10513.

(51) Albacete, P.; Martínez, J. I.; Li, X.; Lopez-Moreno, A.; MenaHernando, S. A.; Platero-Prats, A. E.; Montoro, C.; Loh, K. P.; Perez, E. M.; Zamora, F. Layer-stacking-driven fluorescence in a twodimensional imine-linked covalent organic framework. J. Am. Chem. Soc. 2018, 140, 12922.

(52) Yuasa, J.; Mitsui, A.; Kawai, T. $\pi-\pi^{*}$ Emission from a tetrazine derivative complexed with zinc ion in aqueous solution: a unique water-soluble fluorophore. Chem. Commun. 2011, 47, 5807-5809.

(53) Schwinghammer, K.; Tuffy, B.; Mesch, M. B.; Wirnhier, E.; Martineau, C.; Taulelle, F.; Schnick, W.; Senker, J.; Lotsch, B. V. Triazinebased carbon nitrides for visible-light driven hydrogen evolution. Angew. Chem. Int. Ed. 2013, 52, 2435-2439.

(54) Zhang, J.; Chen, X.; Takanabe, K.; Maeda, K.; Domen, K.; Epping, J. D.; Fu, X.; Antonietti, M.; Wang, X. Synthesis of a carbon nitride structure for visible-light catalysis by copolymerization. Angew. Chem. Int Ed. 2010, 49, 441-444 (2010).

(55) Wang, L.; Fernandez-Teran, R.; Zhang, L.; Fernandes, D. L. A.; Tian, L.; Chen, H.; Tian, H. Organic Polymer Dots as Photocatalysts for Visible Light-Driven Hydrogen Generation. Angew. Chem. Int. Ed. 2016, $55,12306-12310$

(56) Xiang, Y.; Wang, X.; Rao, L.; Wang, P.; Huang, D.; Ding, X.; Zhang X.; Wang, S.; Chen, H.; Zhu, Y. Visible-Light-Driven Photocatalytic Water Oxidation by a $\pi$-Conjugated Donor-Acceptor-Donor Chromophore/Catalyst Assembly. ACS Energy Lett. 2018, 3, 2544-2549.

(57) Chen, W.; Yang, Z.; Xie, Z.; Li, Y.; Yu, X.; Lu, F.; Chen, L. Benzothiadiazole functionalized $\mathrm{D}-\mathrm{A}$ type covalent organic frameworks for effective photocatalytic reduction of aqueous chromium(VI). J. Mater Chem. A, 2019, 7, 998-1004.

(58) Seki, S.; Saeki, A.; Sakurai, T.; Sakamaki, D. Charge carrier mobility in organic molecular materials probed by electromagnetic waves. Phys. Chem. Chem. Phys. 2014, 16, 11093-11113.

(59) Saeki, A.; Koizumi, Y.; Aida, T.; Seki, S. Comprehensive approach to intrinsic charge carrier mobility in conjugated organic molecules, macromolecules, and supramolecular architectures. Acc. Chem Res. 2012, 45, 1193-1202.

(60) Ding, X.; Guo, J.; Feng, X.; Honsho, Y.; Guo, J.; Seki, S.; Maitarad, P.; Saeki, A.; Nagase, S.; Jiang, D. Synthesis of metallophthalocyanine covalent organic frameworks that exhibit high carrier mobility and photoconductivity. Angew. Chem. Int. Ed. $\mathbf{2 0 1 1}$ 50, 1289-1293.

(61) Wan, S.; Gandara, F.; Asano, A.; Furukawa, H.; Saeki, A.; Dey, S. K.; Liao, L.; Ambrogio, M. W.; Botros, Y. Y.; Duan, X.; Seki, S.; Stoddart, J. F.; Yaghi, O. M. Covalent organic frameworks with high charge carrier mobility. Chem. Mater. 2011, 23, 4094-4097.

(62) Ding, X.; Chen, L.; Honsho, Y.; Feng, X.; Saengsawang, O.; Guo, J.; Saeki, A.; Seki, S.; Irle, S.; Nagase, S.; Parasuk, V.; Jiang, D. An n-Channel Two-Dimensional Covalent Organic Framework. J. Am. Chem. Soc 2011, 133, 14510-14513.

(63) Jing, Y.; Heine, T. Two-dimensional kagome lattices made of hetero triangulenes are Dirac semimetals or single-band semiconductors. J. Am. Chem. Soc. 2019, 141, 743-747. 


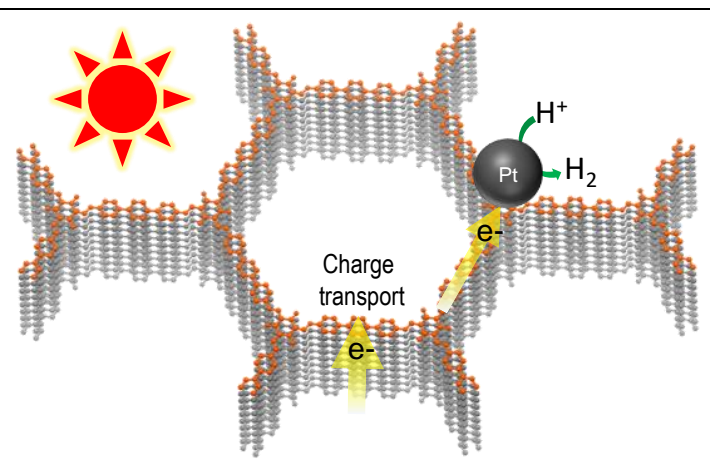

Table of Contents artwork 